Derzeit neun klinische und translationale Forschungsprojekte

Derzeit liefen unter dem YMO-Label fünf klinische Studien und vier translationale Studien bzw. Registerprojekte, berichtete Kruger. Voraussetzung für das Label ist, dass der Studienleiter (LKP) der YMO-Gruppe angehört. Es reicht also nicht, dass YMO an der Studie beteiligt sind. Ein Paradebeispiel für die Arbeit der YMO sei die schon seit einiger Zeit laufende MATEO-Studie, in der an 50 Zentren bei 297 Patienten mit ösophagogastralem Karzinom Erhaltungschemotherapien untersucht werden. Zwei YMO-Studien zum Gallengangskarzinom sind die an der Universität Frankfurt koordinierte IRIBILStudie und die Ulmer NIFE-Studie.

\section{Studien zum NSCLC}

Jonas Kuon von der Thoraxklinik der Universität Heidelberg stellte in Berlin zwei weitere laufende AIO-Studien vor, die sich dem nichtkleinzelligen Lungenkarzinom (NSCLC) widmen. Die FORCE-Studie ist eine Phase-II-Studie, in der untersucht wird, ob sich bei Patienten mit metastasiertem NSCLC nach Versagen der Erstlinienchemotherapie durch eine Strahlentherapie die Effekte einer Immuntherapie verstärken lassen. Hinweise dafür gebe es, so Kuon. Seit Februar 2017 werden daher an 18 Zentren Patienten rekrutiert, die eine bestrahlungspflichtige Tumorläsion aufweisen und bei denen parallel zur Bestrahlung mit der Immuntherapie mit Nivolumab begonnen wird. Als Vergleichsgruppe dienen Patienten, die nur immuntherapeutisch behandelt werden. 130 Patienten sind insgesamt nötig, knapp 30 wurden bereits rekrutiert. Dies zeige, dass die YMO-Gruppe auch Studien mit anspruchsvoller Rekrutierung stemmen könne, so Kuon.

Er selbst leitet unter dem YMO-Label die DURATION-Studie, in der die Immuntherapie in der Zweitlinie bei älteren Patienten mit NSCLC untersucht wird. „Es gibt aus den Zulassungsstudien Hinweise, dass die Immuntherapeutika bei älteren Patienten nicht ganz so effektiv sind. Das wollen wir überprüfen", so Kuon. Konkret erhalten die Patienten in Abhängigkeit von einem Score, mit dem das Toxizitätsrisiko abgeschätzt wird,

\title{
Neue Studienkonzepte der Young Medical Oncologists
}

Von jungen Onkologen wurden bei der Herbsttagung der Arbeitsgemeinschaft Internistische Onkologie (AIO) mehrere neue Studienkonzepte vorgestellt und mit AIO-Vorstandsmitgliedern besprochen. Diese Studien könnten in Zukunft das

Young-Medical-Oncologists(YMO)-Studienlabel der AIO tragen, die Entscheidung darüber ist aber noch nicht in allen Fällen getroffen.

So stellte Silvia Spoerl von der III. Medizinischen Klinik am Klinikum rechts der Isar München eine Studie zur Diskussion, bei der Daten von Patienten mit intestinalem und diffusem Magenkarzinom aufgearbeitet werden sollen. Der intestinale Subtyp habe hinsichtlich des Gesamtüberlebens die bessere Prognose, doch beim diffusen Subtyp werde durch eine neoadjuvante Chemotherapie häufiger eine komplette Remission beobachtet und auch das krankheitsfreie Überleben sei eher besser, so Spoerl. Ziel der Studie ist daher, Patienten mit fortgeschrittenem gastroösophagealem Adenokarzinom von Magen oder Ösophagus beider Subtypen, die eine komplette Remission erreichen, zu vergleichen und detailliert aufzuarbeiten, in Abhängigkeit von der Art der neoadjuvanten Chemotherapie. Dies soll unter anderem mithilfe von Next Generation Sequencing geschehen.

Ein weiterer Projektvorschlag, der im Auftrag von Bernhard Renz von der Chirurgischen Klinik der LMU München vorgestellt wurde, betrifft die unselektive Betablockade in der adjuvanten Therapie von Patienten mit Pankreaskarzinom. Hintergründe sind die bekannt enge Interaktion von Tumorzellen und Nervenzellen beim Pankreaskarzinom, außerdem die Beobachtung, dass eine Betablockade zusätzlich zu einer GemcitabinTherapie in einem Mausmodell für das Pankreaskarzinom das Überleben signifikant verlängerte. Auch in einer Matched-Pair-Analyse von rund 600 Patienten war die unselektive Betablockertherapie mit einem längeren Überleben assoziiert. Daher wurde nun eine klinische Studie vorgeschlagen, um den Zusammenhang auch prospektiv zu überprüfen.

Ebenfalls beim Pankreaskarzinom angesiedelt ist die PROJECTION-Studie, die Benedikt Westphalen vom Krebszentrum der LMU München vorstellte. In dieser geht es um die prognostische Rolle zirkulierender Tumorzellen, die per Liquid Biopsy gewonnen werden sollen. Die Frage lautet, ob eine Liquid Biopsy Aussagen liefern könnte, die verlässlich genug sind, um eventuell eine neoadjuvante bzw. perioperative Chemotherapie zu rechtfertigen. Die Rolle dieser Therapien ist beim Pankreaskarzinom bisher unklar, weil es extrem schwer ist, präoperativ Gewebe für eine definitive Diagnose zu gewinnen, die für eine systemische Chemotherapie aber zu fordern wäre. Philip Grätzel von Grätz

Bericht vom Herbstkongress der Arbeitsgemeinschaft Internistische Onkologie der Deutschen Krebsgesellschaft vom 16. bis 18. November 2017 in Berlin

entweder Carboplatin/nab-Paclitaxel oder Gemcitabin bzw. Vinorelbin. Innerhalb dieser Strata wird dann jeweils randomisiert $\mathrm{zu}$ alleiniger Chemotherapie oder Chemotherapie mit halber Zyklenzahl plus einem Immuntherapeutikum, dem PD-L1 („programmed cell death-ligand 1")-Hemmer Durvalumab.

\section{Junge Onkologen sollten besser repräsentiert sein}

Modest betonte, dass nicht nur die jungen Onkologen von den Strukturen und Erfahrungen der AIO, sondern auch umgekehrt die AIO von den jungen klinischen Forschern profitiere: „Wir bringen zum Beispiel häufiger einen präklinischen Background und damit andere Sichtweisen und andere Kontakte mit.
Auch sind wir weniger eingebunden und haben daher mehr Zeit für die Durchführung von Studien."

Dieses Engagement müsse sich aber auch darin widerspiegeln, dass die YMO in den relevanten Strukturen der AIO angemessen repräsentiert seien. Modest schlug vor, darüber nachzudenken, den LKP-Posten bzw. bei translationalen Studien den Posten des Studienkoordinators generell oder jedenfalls viel häufiger einem YMO zu übertragen. Auch sollten YMO besser als bisher in die Leitgruppen der AIO integriert werden.

Philipp Grätzel von Grätz

Bericht vom Herbstkongress der Arbeitsgemeinschaft Internistische Onkologie der Deutschen Krebsgesellschaft vom 16. bis 18. November 2017 in Berlin 